Research Paper

\title{
Diagnostic value of the 2011 International Federation for Cervical Pathology and Colposcopy Terminology in predicting cervical lesions
}

\author{
Aiping Fan ${ }^{1, *}$, Chen Wang ${ }^{1, *}$, Liqin Zhang ${ }^{1}$, Ye Yan ${ }^{1}$, Cha Han ${ }^{1}$ and Fengxia Xue ${ }^{1}$ \\ ${ }^{1}$ Department of Gynecology and Obstetrics, Tianjin Medical University General Hospital, Tianjin 300052, China \\ *These authors have contributed equally to this work \\ Correspondence to: Aiping Fan, email: fanaipinger@163.com \\ Fengxia Xue, email: fengxiaxue1962@gmail.com \\ Keywords: cervical intraepithelial neoplasia; colposcopy; International Federation for Cervical Pathology and Colposcopy \\ Terminology; squamous intraepithelial lesion; terminology \\ Received: August 31, $2017 \quad$ Accepted: January 02, 2018 Published: January 08, 2018 \\ Copyright: Fan et al. This is an open-access article distributed under the terms of the Creative Commons Attribution License 3.0 \\ (CC BY 3.0), which permits unrestricted use, distribution, and reproduction in any medium, provided the original author and source \\ are credited.
}

\section{ABSTRACT}

Objective: To evaluate the diagnostic accuracy of the 2011 International Federation for Cervical Pathology and Colposcopy (IFCPC) colposcopic terminology. Methods: The clinicopathological data of 2262 patients who underwent colposcopy from September 2012 to September 2016 were reviewed. The colposcopic findings, colposcopic impression, and cervical histopathology of the patients were analyzed. Correlations between variables were evaluated using cervical histopathology as the gold standard.

Results: Colposcopic diagnosis matched biopsy histopathology in 1482 patients $(65.5 \%)$, and the weighted kappa strength of agreement was $0.480(P<0.01)$. Colposcopic diagnoses more often underestimated $(22.1 \%)$ than overestimated $(12.3 \%)$ cervical pathology. There was no significant difference between the colposcopic diagnosis and cervical pathology agreement among the various grades of lesions $(P=0.282)$. The sensitivity, specificity for detecting high-grade lesions/ carcinoma was $\mathbf{7 1 . 6 \%}$ and $\mathbf{9 8 . 0 \%}$, respectively. Multivariate analysis showed that major changes were independent factors in predicting high-grade lesion/carcinoma, whereas transformation zone, lesion size, and non-stained were not statistically related to high-grade lesion/carcinoma.

Conclusions: The 2011 IFCPC terminology can improve the diagnostic accuracy for all lesion severities. The categorization of major changes and minor changes is appropriate. However, colposcopic diagnosis remains unsatisfactory. Poor reproducibility of type 2 transformation zone and the significance of leukoplakia require further study.

\section{INTRODUCTION}

Cervical cancer is the most common malignancy among women in China. Because of the widespread implementation of cervical cancer screening with cervical cytology and/or human papilloma virus (HPV) testing, colposcopy has become more frequently used globally. The use of colposcopy has led to a beneficial reduction in the number of unnecessary blinded biopsies and conization procedures as well as in the frequency of cauterization therapy for cervical erosions. Although colposcopic assessment continues to advance and evolve, universally accepted colposcopic diagnostic criteria and terminology are lacking. Most investigators believe that the traditional Reid colposcopic index (RCI) [1] is simple and easy to use, and it is widely applied. 
Table 1: Agreement between colposcopic diagnoses and cervical histological diagnoses (\%)

\begin{tabular}{lccccc}
\hline \multirow{2}{*}{ Colposcopic diagnosis } & \multicolumn{3}{c}{ Cervical biopsy pathology } & \multirow{2}{*}{ Total } \\
\cline { 2 - 4 } & Normal/benign & LSIL & HSIL & MIC/invasive cancer & \\
\hline Normal/benign & $440(63.7)$ & $290(30.8)$ & $19(3.5)$ & $0(0)$ & 749 \\
LSIL & $244(35.3)$ & $624(66.4)$ & $156(28.7)$ & $4(4.5)$ & 1028 \\
HSIL & $6(0.9)$ & $26(2.8)$ & $366(67.4)$ & $32(36.4)$ & 430 \\
MIC/invasive cancer & $1(0.1)$ & $0(0)$ & $2(0.4)$ & $52(59.1)$ & 55 \\
Total & $691(100)$ & $940(100)$ & $543(100)$ & $88(100)$ & 2262 \\
\hline
\end{tabular}

LSIL, low-grade squamous intraepithelial lesion; HSIL, high-grade squamous intraepithelial lesion; MIC, micro invasive cervical cancer.

However, some scholars believe that the standard method of evaluating color, vessels, and margins of a colposcopic lesion (i.e., grading) to determine the most serious lesion for biopsy has been questioned and traditional grading systems do not accurately assess lesion severity because colposcopic impression alone is unreliable for diagnosis [2]. Most reports have shown a perfect agreement between the colposcopic impression and histology of only $32 \%-37 \%$, with the weighted kappa strength of agreement of only 0.200.26 . The agreement of the colposcopic diagnosis and cervical pathology within one grade has been reported to be only $75 \%-77 \%$, and nearly one - third of highgrade squamous intraepithelial lesions (HSIL) were under graded [3-5]. The goal of clinical management is to identify and treat high-grade disease to decrease the risk of developing invasive cancer. Therefore, the role of colposcopy and biopsy is to identify cervical intraepithelial neoplasia (CIN), especially HSIL. In 2011, the International Federation for Cervical Pathology and Colposcopy (IFCPC) released a new terminology system (the 2011 IFCPC version of the colposcopic terminology, following the 1975, 1990, and 2002 versions of the colposcopic terminology system) and proposed replacing all previous versions of the colposcopic terminology [6]. Although similar to the 2002 version, the new IFCPC terminology is more comprehensive and reclassified some abnormal colposcopic findings on the basis of recent research. In addition, new colposcopic criteria and descriptions of vaginal lesions were added. The aims of the present study were to 1) determine the accuracy of colposcopic diagnosis using the new IFCPC colposcopic terminology; 2) assess the significance of individual colposcopic findings in the new IFCPC colposcopic terminology system; and 3) analyze each specific colposcopic characteristic included in the new IFCPC colposcopic terminology system.

\section{RESULTS}

The data of 2262 patients were included in the analysis. The mean patient age was 41.3 years $(41.3 \pm$ 11.6 years). The agreement of the colposcopic diagnosis and cervical biopsy pathology was perfectly matched in 1482 patients $(65.5 \%)$, and the weighted kappa strength of agreement was $0.480(p<0.01)$. Moreover, agreement within one grade (referring to the same grade or to a difference of only one grade) was observed in 2232 patients $(98.7 \%)$, and the weighted kappa strength of agreement was $0.980(\mathrm{p}<0.01)$. In those patients who were histologically diagnosed as normal or benign, low grade squamous intraepithelial lesions (LSIL), HSIL, or cancer, the agreement between the colposcopy diagnosis and pathological diagnosis was $63.7 \%(440 / 691)$, $66.4 \%$ (624/940), 67.4\% (366/543), and 59.1\% (52/88), respectively. There was no significant difference among the four groups $\left(\chi^{2}=3.813, \mathrm{p}=0.282\right)$. The colposcopic diagnosis was overestimated in 279 patients $(12.3 \%$, $279 / 2262)$ and underestimated in 501 patients $(22.1 \%$, $501 / 2262$ ). Within two grades (referring to the same grade or to a difference of two grades) or more, 7 patients $(0.3 \%, 7 / 2262)$ were overestimated and 23 patients $(1.0 \%$, 23/2262) were underestimated (Table 1). The accuracy of colposcopy for the detection of LSIL or more (LSIL+) and HSIL or more (HSIL+) is shown in Table 2.

Of the 2262 patients, 340 patients underwent conization or hysterectomy. The agreement of the colposcopic diagnosis and final histopathologic diagnosis was perfectly matched in 225 patients (66.2\%) (Table 3 ). In those patients who were histologically diagnosed as normal or benign, LSIL, HSIL, or cancer, the agreement between the colposcopy diagnosis and final pathological diagnosis was $100.0 \%(0 / 0), 72.7 \%(8 / 11), 68.9 \%$ $(199 / 289)$, and $45.0 \%(18 / 40)$, respectively. There was a significant difference among the four groups $\left(\chi^{2}=9.153\right.$, $p$ $=0.010)$. Although there is no colposcopic overestimate, 115 patients $(33.8 \%, 115 / 340)$ were underestimated. 
Table 2: Accuracy of colposcopic diagnosis in distinguishing cervical pathology at different cutoffs

Cutoff for colposcopic diagnosis

\begin{tabular}{|c|c|c|}
\hline & \\
\hline & $\begin{array}{c}\text { Any lesion } \\
\text { (low-grade/high-grade/carcinoma) } \\
\text { vs. healthy cervix }\end{array}$ & $\begin{array}{c}\text { High-grade } \\
\text { lesion/carcinoma vs. } \\
\text { healthy cervix/low-grade lesion }\end{array}$ \\
\hline Sensitivity $(95 \% \mathrm{CI})$ & $80.3 \%(78.4-82.3 \%)$ & $71.6 \%(68.1 \%-75.1 \%)$ \\
\hline Specificity $(95 \% \mathrm{CI})$ & $63.7 \%(60.1 \%-67.3 \%)$ & $98.0 \%(97.3 \%-98.7 \%)$ \\
\hline Positive predictive value $(95 \% \mathrm{CI})$ & $83.4 \%(81.5 \%-85.3 \%)$ & $93.2 \%(91.0 \%-95.4 \%)$ \\
\hline Negative predictive value $(95 \% \mathrm{CI})$ & $58.7 \%(55.2 \%-62.3 \%)$ & $89.9 \%(88.5 \%-91.3 \%)$ \\
\hline Youden index $(95 \% \mathrm{CI})$ & $44.0 \%(39.9 \%-48.1 \%)$ & $69.6 \%(66.0 \%-73.2 \%)$ \\
\hline
\end{tabular}

Table 3: Agreement between colposcopic diagnoses and final histological diagnoses (\%)

\begin{tabular}{lccccc}
\hline & \multicolumn{3}{c}{ Final histological diagnosis } & Total \\
\cline { 2 - 5 } Colposcopic diagnosis & Normal/benign & LSIL & HSIL & $\begin{array}{c}\text { MIC/invasive } \\
\text { cancer }\end{array}$ \\
\hline Normal/benign & $0(0)$ & $3(27.3)$ & $4(1.4)$ & $0(0)$ & 7 \\
LSIL & $0(0)$ & $8(72.7)$ & $86(29.7)$ & $2(5.0)$ & 96 \\
HSIL & $0(0)$ & $0(0)$ & $199(68.9)$ & $20(50.0)$ & 219 \\
MIC/invasive cancer & $0(0)$ & $0(0)$ & $0(0)$ & $18(45.0)$ & 18 \\
Total & $0(0)$ & $11(100)$ & $289(100)$ & $40(100)$ \\
\hline
\end{tabular}

LSIL, low-grade squamous intraepithelial lesion; HSIL, high-grade squamous intraepithelial lesion; MIC, micro invasive cervical cancer.

Overall, 1005 cases $(44.4 \%, 1005 / 2262)$ were classified as type 2 transformation zone (TZ), 887 (39.2\%, $887 / 2262)$ as type $1 \mathrm{TZ}$, and 370 (16.4\%, 370/2262) as type $3 \mathrm{TZ}$. Among 370 cases classified as type $3 \mathrm{TZ}$, the age of 243 patients $(65.7 \%, 243 / 370)$ was $>50$ years, whereas the frequency of this TZ type was low in women aged $30-50$ years $(31.9 \%, 118 / 370)$ and rare in those aged $<30$ years $(2.4 \%, 9 / 370)$. The most frequently reported TZ type in women aged $<30$ years was type $1(70.6 \%$, 283/401). Among 1005 cases classified as type 2 TZ, 665 patients $(66.2 \%, 665 / 1005)$ were aged $30-50$ years. The linear trend test showed a correlation between TZ types and the three age groups $\left(\chi^{2}=534.324, \mathrm{p}<0.01\right)$.

Of 2262 patients, 1955 patients demonstrated abnormal colposcopic findings on $<30 \%$ of the visible cervix $(86.4 \%, 1955 / 2262), 148$ patients had colposcopic abnormalities on $30 \%-67 \%$ of the visible cervix $(6.5 \%$, $148 / 2262$ ), and 159 patients had abnormalities on $>67 \%$ of the visible cervix $(7.0 \%, 159 / 2262)$. The linear trend test showed a correlation between the size of the lesion and pathological diagnosis: the larger the size of the lesion, the more serious the disease $\left(\chi^{2}=466.107, \mathrm{p}<0.01\right)$.

Correlation between abnormal colposcopic findings and cervical histological diagnoses is shown in Table 4. With regard to minor changes, the incidence of a thin acetowhite epithelium was the highest $(65.4 \%$, $1479 / 2262)$, followed by fine punctation $(48.5 \%$, $1096 / 2262)$ and fine mosaic $(37.2 \%, 842 / 2262)$. The sensitivity, specificity, positive predictive value (PPV), negative predictive value (NPV), and Youden index for the detection of LSIL are shown in Table 5.

Colposcopic findings graded as major changes had a high predictive value for HSIL/carcinoma. The sensitivity, specificity, PPV, NPV, and Youden index for the detection of HSIL+ for major changes are shown in Table 6. The two new colposcopic criteria (inner border sign and ridge sign) also had a high predictive value for HSIL+. The specificity and PPV of the inner border sign for HSIL+ was $99.6 \%$ (1624/1631) and 75.0\% (21/28), respectively. The specificity and PPV of the ridge sign for HSIL+ was 99.4\% (1622/1631) and 91.4\% (96/105), respectively.

Of the non-specific signs, non-stained lesions were very common $(85.8 \%, 1940 / 2262)$ and had a high sensitivity and NPV, but it was unsatisfactory for the specificity, PPV and Youden index. Although iodinestained lesions had a high specificity, PPV and NPV, the sensitivity was very low (27.3\%). Among 15 patients $(0.7 \%, 15 / 2262)$ with leukoplakia, LSIL was found in 
Table 4: Correlation between colposcopic findings and cervical histological diagnoses (\%)

\begin{tabular}{|c|c|c|c|c|c|}
\hline \multirow[b]{2}{*}{ Colposcopic finding } & \multicolumn{4}{|c|}{ Cervical biopsy pathology } & \multirow[b]{2}{*}{ Total } \\
\hline & Normal/benign & LSIL & HSIL & $\begin{array}{l}\text { MIC/invasive } \\
\text { cancer }\end{array}$ & \\
\hline \multicolumn{6}{|l|}{ Minor changes } \\
\hline Thin acetowhite epithelium & $518(35.0))$ & $790(53.4)$ & $168(11.4)$ & $3(0.2)$ & $1479(100)$ \\
\hline Fine mosaic & $210(24.9)$ & $406(48.3)$ & $220(26.1)$ & $6(0.7)$ & $842(100)$ \\
\hline Fine punctation & $337(30.8)$ & $559(51.0)$ & 193(17.6) & $7(0.6)$ & $1096(100)$ \\
\hline \multicolumn{6}{|l|}{ Major changes } \\
\hline Dense acetowhite epithelium & $18(3.3)$ & $68(12.6)$ & $374(69.0)$ & $82(15.1)$ & $542(100)$ \\
\hline Coarse mosaic & $0(0)$ & $4(2.4)$ & 131(79.9) & $29(17.7)$ & $164(100)$ \\
\hline Coarse punctation & $1(0.4)$ & $18(7.5)$ & $186(77.2)$ & $36(14.9)$ & 241(100) \\
\hline Sharp border & $3(6.2)$ & $2(4.2)$ & $31(64.6)$ & $12(25.0)$ & $48(100)$ \\
\hline Inner border sign & $0(0)$ & $7(25.0)$ & $19(67.9)$ & $2(7.1)$ & $28(100)$ \\
\hline Ridge sign & $2(1.9)$ & $7(6.7)$ & $77(73.3)$ & $19(18.1)$ & $105(100)$ \\
\hline \multicolumn{6}{|l|}{ Non specific } \\
\hline Non-stained & $523(27.0)$ & $808(41.6)$ & $522(26.9)$ & $87(4.5)$ & $1940(100)$ \\
\hline Leukoplakia & $3(20.0)$ & $4(26.7)$ & $8(53.3)$ & $0(0)$ & $15(100)$ \\
\hline \multicolumn{6}{|l|}{ Suspicious for invasion } \\
\hline Atypical vessels & $1(1.3)$ & $1(1.3)$ & $21(26.5)$ & $56(70.9)$ & $79(100)$ \\
\hline \multicolumn{6}{|l|}{ Miscellaneous finding } \\
\hline condyloma & $7(18.9)$ & $26(70.3)$ & $4(10.8)$ & $0(0)$ & $37(100)$ \\
\hline polyp & $93(95.8)$ & $2(2.1)$ & $2(2.1)$ & $0(0)$ & $97(100)$ \\
\hline
\end{tabular}

LSIL, low-grade squamous intraepithelial lesion; HSIL, high-grade squamous intraepithelial lesion; MIC, micro invasive cervical cancer.

Table 5: Accuracy of colposcopic minor changes in predicting low-grade lesion

\begin{tabular}{|c|c|c|c|c|c|}
\hline Minor changes & $\begin{array}{l}\text { Sensitivity } \\
(95 \% \text { CI) }\end{array}$ & $\begin{array}{l}\text { Specificity } \\
(95 \% \text { CI) }\end{array}$ & $\begin{array}{l}\text { Positive predictive value } \\
\text { (95\% CI) }\end{array}$ & $\begin{array}{l}\text { Negative predictive value } \\
\qquad(95 \% \mathrm{CI})\end{array}$ & $\begin{array}{c}\text { Youden index } \\
(95 \% \% \mathrm{CI})\end{array}$ \\
\hline $\begin{array}{l}\text { Thin aceto-white } \\
\text { epithelium }\end{array}$ & $84.0 \%(81.7 \%-86.4 \%)$ & $47.9 \%(45.2 \%-50.6 \%)$ & $53.4 \%(50.9 \%-56.0 \%)$ & $80.8 \%(78.1 \%-83.6 \%)$ & $31.9 \%(28.4 \%-35.5 \%)$ \\
\hline Fine mosaic & $43.2 \%(40.0 \%-46.4 \%)$ & $67.0 \%(64.5 \%-69.5 \%)$ & $48.2 \%(44.8 \%-51.6 \%)$ & $62.4 \%(59.9 \%-64.9 \%)$ & $10.2 \%(6.2 \%-14.3 \%)$ \\
\hline Fine punctation & $59.5 \%(56.3 \%-62.6 \%)$ & $59.4 \%(56.7 \%-62.0 \%)$ & $51.0 \%(48.0 \%-54.0 \%)$ & $67.3 \%(64.6 \%-70.0 \%)$ & $18.8 \%(14.7 \%-23.0 \%)$ \\
\hline
\end{tabular}

4 patients, and HSIL in 8 patients. Leukoplakia had a considerable PPV for HSIL $(53.3 \%, 8 / 15)$.

Invasive cancers were rare and found in only 88 patients $(3.9 \%, 88 / 2262)$. However, some colposcopic findings classified as suspicious for invasion, such as atypical vessels had a considerable PPV for HSIL+ $(97.5 \%, 77 / 79)$.

Several colposcopic findings classified as miscellaneous findings had a low PPV (condyloma: 10.8\%, 4/37; polyp: 2.1\%, 2/97) for HSIL+. Among 37 patients with condyloma, LSIL was found in 26 patients, and HSIL in 4 patients. Among 97 patients with a polyp, LSIL was found in 2 patients, and HSIL in only 2 patients. Some colposcopic findings in predicting LSIL/ HSIL+ are summarized in Tables 7 and 8. On the basis of univariate analysis, factors such as TZ type ( $\mathrm{p}=0.019$ and $\mathrm{p}=0.001$ for LSIL and HSIL + , respectively), lesion size $(\mathrm{p}=0.002$ and $\mathrm{p}=0.000$ for LSIL and HSIL+, respectively), colposcopic minor/major changes (both $\mathrm{p}=0.000$ ), and no-stained lesions (both $\mathrm{p}=0.000$ ) were all linked with the 
Table 6: Accuracy of colposcopic major changes in predicting high-grade lesion/carcinoma

\begin{tabular}{|c|c|c|c|c|c|}
\hline Major changes & $\begin{array}{l}\text { Sensitivity } \\
\text { (95\% CI) }\end{array}$ & $\begin{array}{l}\text { Specificity } \\
\text { (95\% CI) }\end{array}$ & $\begin{array}{c}\text { Positive predictive value } \\
\text { (95\% CI) }\end{array}$ & $\begin{array}{l}\text { Negative predictive value } \\
(95 \% \mathrm{CI})\end{array}$ & $\begin{array}{l}\text { Youden index } \\
(95 \% \mathrm{CI})\end{array}$ \\
\hline $\begin{array}{l}\text { Dense aceto-white } \\
\text { epithelium }\end{array}$ & $72.3 \%(68.6 \%-75.7 \%)$ & $94.7 \%(93.5 \%-95.8)$ & $84.1 \%(80.8 \%-87.1 \%)$ & $89.8 \%(88.3 \%-91.2 \%)$ & $67.0 \%(63.3 \%-70.7 \%)$ \\
\hline Coarse punctation & $35.2 \%(31.5 \%-39.1 \%)$ & $98.8 \%(98.2 \%-99.3 \%)$ & $92.1 \%(88.0 \%-95.2 \%)$ & $79.8 \%(77.9 \%-81.5 \%)$ & $34.0 \%(30.3 \%-37.8 \%)$ \\
\hline Coarse mosaic & $25.4 \%(22.0 \%-28.9 \%)$ & $99.8 \%(99.4 \%-99.9 \%)$ & $97.6 \%(93.9 \%-99.3 \%)$ & $77.6 \%(75.7 \%-79.3 \%)$ & $25.1 \%(21.7 \%-28.5 \%)$ \\
\hline Sharp border & $6.8 \%(4.8 \%-8.8 \%)$ & $99.7 \%(99.4 \%-100 \%)$ & $89.6 \%(80.9 \%-98.2 \%)$ & $73.4 \%(71.6 \%-75.3 \%)$ & $6.5 \%(4.5 \%-8.5 \%)$ \\
\hline Ridge sign & $15.2 \%(12.5 \%-18.3 \%)$ & $99.4 \%(99.0 \%-99.7 \%)$ & $91.4 \%(84.4 \%-96.0 \%)$ & $75.2 \%(73.3 \%-77.0 \%)$ & $14.7 \%(11.7 \%-17.5 \%)$ \\
\hline Inner border sign & $3.3 \%(2.1 \%-5.0 \%)$ & $99.6 \%(99.1 \%-99.8 \%)$ & $75.0 \%(55.1 \%-89.3 \%)$ & $72.7 \%(70.8 \%-74.5 \%)$ & $2.9 \%(1.5 \%-4.3 \%)$ \\
\hline
\end{tabular}

Table 7: Colposcopic findings in predicting low-grade lesion

\begin{tabular}{|c|c|c|c|c|c|}
\hline Colposcopic findings & $\mathbf{n}$ & $\begin{array}{c}\text { Discordant } \\
(n=317)\end{array}$ & $\begin{array}{l}\text { Concordant } \\
(n=623)\end{array}$ & $\begin{array}{l}\text { Univariate analysis } \\
\text { OR }(95 \% \mathrm{CI}) / \mathbf{p}\end{array}$ & $\begin{array}{c}\text { Binary logistic regression } \\
\text { OR }(95 \% \mathrm{CI}) / \mathrm{p}\end{array}$ \\
\hline Transformation zone type & & & & $0.782(0.637-0.960) / 0.019$ & $1.161(0.920-1.465) / 0.209$ \\
\hline 1 & 424 & 134 & 290 & & \\
\hline 2 & 422 & 138 & 284 & & \\
\hline 3 & 94 & 45 & 49 & & \\
\hline \multicolumn{4}{|c|}{ Percentage of cervix encompassed by the lesion } & $2.775(1.446-5.329) / 0.002$ & $2.047(1.049-3.991) / 0.036$ \\
\hline$<30 \%$ & 887 & 312 & 575 & & \\
\hline $30 \% \sim 67 \%$ & 34 & 2 & 32 & & \\
\hline$>67 \%$ & 19 & 3 & 16 & & \\
\hline Minor changes & & & & $3.083(2.506-3.793) / 0.000$ & $3.145(2.505-3.949) / 0.000$ \\
\hline$\leq 1$ & 233 & 144 & 89 & & \\
\hline$\geq 2$ & 707 & 173 & 534 & & \\
\hline Non-stained lesions & & & & $0.390(0.269-0.566) / 0.000$ & $0.658(0.432-1.002) / 0.051$ \\
\hline Yes & 808 & 247 & 561 & & \\
\hline No & 132 & 70 & 62 & & \\
\hline Leukoplakia & & & & $827311037.174(0.000-\infty) / 0.999$ & $2379464167.734(0.000-\infty) / 0.999$ \\
\hline Yes & 4 & 0 & 4 & & \\
\hline No & 936 & 317 & 619 & & \\
\hline
\end{tabular}

diagnostic accuracy of colposcopy for predicting LSIL and HSIL + . However, leukoplakia ( $p=0.999$ and $p=0.822$ for LSIL and HSIL+, respectively) did not correlate with the diagnostic accuracy of colposcopy for predicting LSIL and HSIL +. On the basis of multivariate analysis with binary logistic regression, lesion size $(p=0.036)$ and colposcopic minor changes $(p=0.000)$ were significant independent factors for predicting LSIL, and only colposcopic major changes $(p=0.000)$ were a significant independent factor in predicting HSIL/carcinoma.

We also analyzed HPV genotyping data. Of the 2262 patients, 62 were not tested for HPV, 284 were negative for HPV, 23 were positive for Hybrid Capture II test, and 1893 were positive for HPV genotyping test.
Single high-risk HPV (HR-HPV) infections were found in 993 of 1893 patients (52.4\%), single low-risk HPV (LR-HPV) infections were found in 72 of 1893 patients (3.8\%), and multiple infections ( multiple HR-HPV infections, 440 cases; HR-HPV+ LR-HPV multiple infections, 375 cases; LR-HPV multiple infections, 13 cases) were present in 828 of 1893 positive samples (43.7\%). Most of the multiple infections consisted of two HPV types $(58.9 \%, 488 / 828)$, whereas the rest had three to eight HPV types. A high prevalence of single HR-HPV infection was found among HSIL (56.1\%, 291/519) and micro invasive cervical cancer (MIC) / invasive cancer $(66.7 \%, 54 / 81)$. Significant differences $\left(\chi^{2}=19.935\right.$, $\mathrm{p}=0.000)$ between single HR-HPV infections and multiple 
Table 8: Colposcopic findings in predicting high-grade lesion/carcinoma

\begin{tabular}{|c|c|c|c|c|c|}
\hline Colposcopic findings & $\mathbf{n}$ & $\begin{array}{l}\text { Discordant } \\
(n=213)\end{array}$ & $\begin{array}{l}\text { Concordant } \\
(n=418)\end{array}$ & $\begin{array}{c}\text { Univariate analysis } \\
\text { OR }(95 \% \text { CI }) / p\end{array}$ & $\begin{array}{c}\text { Binary logistic regression } \\
\text { OR }(95 \% \text { CI }) / p\end{array}$ \\
\hline \multicolumn{4}{|l|}{ Transformation zone type } & $1.478(1.166-1.873) / 0.001$ & $1.347(0.992-1.828) / 0.056$ \\
\hline 1 & 178 & 75 & 103 & & \\
\hline 2 & 309 & 102 & 207 & & \\
\hline 3 & 144 & 36 & 108 & & \\
\hline \multicolumn{4}{|c|}{ Percentage of cervix encompassed by the lesion } & $2.571(1.976-3.345) / 0.000$ & $1.079(0.781-1.489) / 0.645$ \\
\hline$<30 \%$ & 391 & 176 & 215 & & \\
\hline $30 \% \sim 67 \%$ & 104 & 20 & 84 & & \\
\hline$>67 \%$ & 136 & 17 & 119 & & \\
\hline Major changes & & & & $5.373(4.144-6.965) / 0.000$ & $5.070(3.853-6.673) / 0.000$ \\
\hline$\leq 1$ & 319 & 182 & 137 & & \\
\hline$\geq 2$ & 312 & 31 & 281 & & \\
\hline Non-stained lesions & & & & $0.179(0.069-0.465) / 0.000$ & $0.383(0.103-1.428) / 0.153$ \\
\hline Yes & 609 & 197 & 412 & & \\
\hline No & 22 & 16 & 6 & & \\
\hline Leukoplakia & & & & $0.847(0.201-3.580) / 0.822$ & $1.012(0.164-6.251) / 0.990$ \\
\hline Yes & 8 & 3 & 5 & & \\
\hline No & 623 & 210 & 413 & & \\
\hline
\end{tabular}

HR-HPV infections were observed regarding the histology findings on colposcopically directed biopsy. HPV Type 16 was identified most frequently $(33.9 \%, 642 / 1893)$, followed by types $52(17.0 \%, 321 / 1893), 58(16.1 \%$, 304/1893), 56(9.2\%, 174/1893), 33(8.7\%, 164/1893), and $18(8.6 \%, 162 / 1893)$. The prevalence of HPV type 16 increased with the grade of cervical disease, as defined by biopsy-histological diagnosis: 18.2\% (167/910) in LSIL, $51.6 \%(268 / 519)$ in HSIL, and 81.5\% (66/81) in cervical carcinoma. On the basis of univariate analysis (HR, 0.567 ; $95 \% \mathrm{CI}, 0.397-0.808 ; \mathrm{p}=0.002)$ and multivariate analysis (HR, 0.667; 95\%CI, 0.454-0.979; $\mathrm{p}=0.039$ ), the presence of HPV type 16 was correlated with cervical HSIL+, and it was an independent predictor factor for cervical HSIL+ development. Although HPV types 18, 58, 52, and 33 were also identified frequently in HSIL + , presence of HPV types $18,58,52$, and 33 was not associated with this risk.

\section{DISCUSSION}

Colposcopic examination involves the systematic evaluation of the lower genital tract. The diagnostic criteria for colposcopy continue to improve and evolve [1, $6,9,10]$. However, the literature regarding the accuracy of colposcopy is contradictory, and no uniform standard has been accepted. The correlation between the colposcopic impression and histology remains poor [11-14].
We analyzed the applicability of the new IFCPC colposcopic terminology for predicting cervical lesions. The study results showed that the agreement of the colposcopic diagnosis and cervical biopsy pathology was perfectly matched in $65.5 \%$ of lesions, further, the agreement of the colposcopic diagnosis and final pathology was perfectly matched in $66.2 \%$ of lesions, with findings significantly better than those of Baum et al. and Massad et al (perfect agreement, 32-37\%) $[3,4]$. Tatiyachonwiphut et al. [13] reported an exact agreement of $57.9 \%$, one grade agreement of $94.1 \%$, and a weighted kappa strength of agreement of 0.494 , which were comparable to the results of our study. However, the study lacked a standard criterion or scoring system for colposcopic examination. Li et al. [14], using the 2011 IFCPC colposcopic terminology, reported findings comparable to those of our study; however, their sample size was small. Mousavi et al. and Durdi et al. [11, 15] reported a higher rate of perfect agreement $(77.3 \%$ and $85.8 \%$, respectively) and a substantial level of correlation ( $\kappa=0.74$ and $\kappa=0.73$, respectively) using the RCI scoring system, but the sample sizes were small and HR-HPV testing was not done. In addition, the RCI method depends on the severity of disease, and the predictive accuracy increases with the advancing severity of the lesion. Our study showed that there were no significant differences between the colposcopic diagnosis and cervical pathology 
agreement among the various grades of lesions $(\mathrm{P}=$ 0.282 ), further suggesting that the accuracy of colposcopic diagnosis using the IFCPC terminology was stable and did not vary with lesion severity.

In the present study, the colposcopic diagnosis more frequently underestimated $(22.1 \%)$ than overestimated $(12.3 \%)$ the cervical biopsy pathology. For those patients who underwent conization or hysterectomy, although there is no colposcopic overestimate, 115 patients $(33.8 \%, 115 / 340)$ were underestimated. An overestimated colposcopic diagnosis leads to an unnecessary cervical biopsy, whereas an underestimated diagnosis leads to missed diagnoses, possibly high-grade lesions/carcinomas. The primary task for colposcopists is to improve the accuracy of colposcopic diagnosing HSIL+ and prevent the growth of undiagnosed lesions.

In the present study, we found that the colposcopic examination has a high sensitivity $(80.3 \%)$ and is capable of differentiating a healthy cervix from any cervical lesion in $80.3 \%$ of the cases; however, the specificity was low $(63.7 \%)$, probably leading to over-examination and thereby increased patients cost. Some studies have reported that such over-examination causes the patient to undergo an unnecessary cervical biopsy. We also found that colposcopy had a $71.6 \%$ sensitivity for the detection of HSIL+, similar to that reported in previous studies $(30 \%$ 91.3\%) [9, 13-17]. Moreover, the specificity for detecting HSIL was high (98.0\%), also comparable to that previously reported (79\% - 96.5\%) [13-15]. A high specificity lowers the misdiagnosis rate, which is important with regard to clinical management because of the significant differences in the clinical management of LSIL and HSIL. The PPV of colposcopy to diagnose HSIL+ was $93.2 \%$, which is also comparable to the findings of previous reports [9, 13-15]. The NPV of HSIL was $89.9 \%$, again comparable with previous studies (83.8\%-93.5\%) [13-15]. On the basis of these findings, if a cervical biopsy specimen was obtained from every abnormal area detected during colposcopy, $28.4 \%$ of patients would benefit from early detection and treatment of HSIL+, and only $2.0 \%$ of patients would have undergone an unnecessary cervical biopsy.

Precise identification of the squamocolumnar junction and evaluation of the $\mathrm{TZ}$ are crucial steps during colposcopy. The 2011 IFCPC colposcopic terminology confirmed the classification of the $\mathrm{TZ}$ as an obligatory terminology. Our study showed a linear trend between the TZ type and three age groups, with type $3 \mathrm{TZ}$ found more commonly in women $>50$ years old and type $1 \mathrm{TZ}$ found more frequently in women $<30$ years old. Further, in the present study, univariate analysis revealed that TZ type was linked to the diagnostic accuracy of colposcopy. However, binary logistic regression analysis revealed that TZ type was not an independent predictor factor in diagnosing LSIL or HSIL/carcinoma. A prospective multicenter study by Luyten et al. compared the distribution of $\mathrm{TZ}$ types among women according to age [18]. In their study, type 3 TZ was most commonly reported in women $>50$ years old (70\%). However, there was evidence of a heterogeneous distribution of TZ types 1 and 2 between the age groups, possibly because of a diagnostic uncertainty between these types. They proposed a more precise anatomic distinction between types 1 and 2 in the IFCPC terminology to improve the utility of the new IFCPC classification and to encourage standardized universal reporting of findings. However, the 2017 American Society for Colposcopy and Cervical Pathology (ASCCP) colposcopy standards [19] did not divide cervical TZ into types 1,2 , and 3 , mainly because of the poor reproducibility of type $2 \mathrm{TZ}$ in actual clinical work. With regard to cervical TZ, more clinical studies are needed to confirm its feasibility and clinical value in colposcopic examination.

Lesion size has also been included in the new terminology system. Our study revealed a linear trend between lesion size and severity of the diagnosis, and a larger lesion size was more closely related to HSIL+. However, binary logistic regression analysis revealed that lesion size was not an independent predictor factor in diagnosing HSIL + . Several previous studies have shown that a large lesion size was more closely related to HSIL or more, and a small lesion size predicted the absence of a squamous epithelial lesion/CIN [17, 20, 21].

The 2011 IFCPC terminology has revised the grading nomenclature by adding two new pathognomonic, objective colposcopic criteria: the "inner border sign" and "ridge sign". We analyzed these specific signs in detail, and the specificity and PPV of both signs were high. Other studies have confirmed the clinical utility of these criteria and their strong association with HSIL [22-25]. With regard to other minor changes and major changes, the incidence of a thin acetowhite epithelium was the highest (65.4\%). However, a thin acetowhite epithelium was found in only $27.1 \%$ of cases with HSIL, and had little or no association with HSIL+. Some studies have reported that a thin acetowhite epithelium does not suggest the existence of a cervical vaginal lesion [17]; however, other studies have reported a thin acetowhite epithelium with HSIL in an atrophic area [26]. Massad et al. proposed that all acetowhite lesions should be biopsied to maximize the sensitivity of colposcopic diagnosis while maintaining a good specificity [16]. In the present study, we found that two or more colposcopic minor changes were independent predictor factors in diagnosing LSIL. Additionally, we also found that a dense aceto-white epithelium, coarse mosaic, and coarse punctation had a high specificity and PPV for HSIL+ and were significantly associated with severe cervical lesions, and two or more colposcopic major changes were independent predictor factors in diagnosing HSIL+. These findings further confirm that the categorization of major changes and minor changes is appropriate. 
Another common sign, a non-stained lesion, was once classified as a major change in the RCI scoring system and previous IFCPC colposcopic terminology. However, non-stained lesions are currently classified as a non-specific sign in the 2011 IFCPC terminology. Our study further confirmed that the diagnostic value of nonstained lesions for HSIL+ is very low, and was not an independent predictor factor for HSIL+; although iodinestained lesions had a high specificity, PPV, and NPV, the sensitivity was very low. Leukoplakia had a considerable PPV of 53.3\% for HSIL+; however, our data revealed that leukoplakia was not an independent predictor factor for HSIL+. The present published studies are discordant on the significance of this terminology. In addition, it is worth noting that 30 of 37 cases suggestive of condyloma were related to LSIL/HSIL, suggesting that we should pay close attention to cervical condyloma during colposcopy, and that cervical biopsy should be performed if indicated.

High risk HPV- related infections are a significant source of cervical morbidity and mortality. Our data revealed that HPV type 16 was identified most frequently, followed by HPV types 52, 58, 56, 33, and 18. The prevalence of HPV type 16 increased with the grade of the cervical disease as defined by biopsy-histological diagnosis, and the presence of HPV type 16 was an independent factor for predicting cervical HSIL+ development, whereas the presence of HPV types 18, $33,52,56$, and 58 were not associated with this risk. This implies that HR-HPV infections, especially HPV type 16 , increase the risk of developing severe lesions. As colposcopy is inadequate for patients or in the case of suspected cervical HSIL+, colposcopic examination should be combined with cervical cytology and HRHPV genotyping, especially with HPV type 16, to further improve diagnostic accuracy.

Our study had several limitations. First, the study design was retrospective in nature and enrollment bias may have occurred. Second, colposcopy diagnosis may have been biased by prior knowledge of the cervical cytology results. Third, although the conization/hysterectomy data had been used to strengthen the pathology diagnosis, the sample size is less. Finally, we did not compare the overall diagnostic accuracy of 2011 IFCPC criteria with that of other colposcopic diagnostic criteria (such as RCI) for the detection of high-grade CIN.

The clinical application of the 2011 IFCPC terminology is in its preliminary stage. Our data revealed that the categorization of major changes and minor changes is appropriate; the accuracy of colposcopic diagnosis using the IFCPC terminology was stable and did not vary with lesion severity. However, there was only a moderate strength of agreement between colposcopic diagnosis and cervical pathology, and the diagnostic accuracy remains unsatisfactory. Therefore, we propose that colposcopic findings should be combined with cervical cytology and HR-HPV genotyping test, especially with HPV type 16, to further improve the diagnostic accuracy. Studies evaluating the 2011 IFCPC colposcopic terminology are limited and further prospective studies are needed. In future studies, we aim to investigate RCI and 2011 IFCPC criteria and obtain data with respect to accuracy of the two colposcopic diagnostic methods.

\section{MATERIALS AND METHODS}

\section{Subjects}

We retrospectively reviewed the medical records of women who underwent colposcopic examinations and cervical biopsy at the colposcopy clinic of the General Hospital of Tianjin Medical University between September 2012 and September 2016. These women had no previous surgical procedures of the cervix, no history of pelvic radiation or hysterectomy, no sexual intercourse for 3 days before the examination, and no confirmed or clinically suspected immunosuppression (e.g., HIV, corticosteroids, chemotherapy) or other chronic disease that might have compromised their immune system. Colposcopy indications included atypical or abnormal cervical cytology results and/ or positivity for HR-HPV and/or suspicious clinical findings. Positive cervical cytology included atypical squamous cells of uncertain significance; atypical squamous cell, cannot exclude HSIL; LSIL; HSIL; atypical glandular cells; and invasive cervical cancer. HPV genotyping was performed via the PCR-reverse dot hybridization method. Eighteen HR-HPV types $(16,18$, $31,33,35,39,45,51,52,53,56,58,59,66,68,73,82$, and 83 ) and five LR-HPV types $(6,11,42,43$, and 81$)$ were identified. The data in this study were collected from the hospital's archived database. This study did not influence the diagnosis or treatment of the patients. Informed consent was waived by the ethics review board of the General Hospital of Tianjin Medical University. The institutional review board approved this (No. IRB 2016-YX-073).

\section{Colposcopy and biopsy procedure}

At the colposcopy clinic, all colposcopies were performed by two expert colposcopists with more than five years of experience who had received the ASCCP professional colposcopy training. All colposcopic examinations were performed and recorded using the VIZ-YD system, which is a video exoscope-based system (optical electronic integration colposcopy, Beijing SWSY technology Co., Ltd, China) that allows full HD video documentation of the colposcopic examination and is used for clinical records and research. Steps in colposcopic assessment of the cervix included the following: 1) clean the cervix with normal saline; 2) assess the cervix about $1 \mathrm{~min}$ after the application of $5 \%$ 
acetic acid, and observe margins of the lesion, epithelial color and vascular patterns; and 3) assess the cervix after the application of diluted Lugol's iodine solution, and observe iodine staining. Colposcopic findings were described using the 2011 IFCPC terminology: (1) TZ types, lesion size in percentage of cervix, and normal colposcopic findings (such as original squamous epithelium: mature or atrophic, columnar epithelium ectopy, Nabothian cysts, crypt [gland] openings); (2) abnormal colposcopic findings, grade 1( minor) (thin acetowhite epithelium[irregular, geographic, border], fine punctation, fine mosaic), grade 2 (major) (dense acetowhite epithelium, rapid appearance of acetowhitening, cuffed crypt [gland] openings, coarse punctation, coarse mosaic, sharp border, inner border sign, ridge sign), non specific (Leukoplakia [keratosis, hyperkeratosis], Erosion, stained/non-stained Lugol's staining [Schiller's test]); (3) suspicious for invasion, atypical vessels, additional signs; and (4) miscellaneous findings, such as condyloma, polyp, inflammation, and post treatment consequence. It is impossible that all abnormal findings (suggestive of the same grade disease) simultaneously exist in one patient. As a result, colposcopists made a preliminary colposcopic diagnosis for each colposcopic finding on the basis of the criteria of the IFCPC. It should be noted that the same colposcopic finding could be present in different grades of lesions. When doubtful diagnosis occurred, all the inconsistent diagnoses were analyzed by another senior colposcopist, who made a decision on the final colposcopic diagnosis.

All colposcopically detected abnormal areas were biopsied, and large or multiple lesions were subjected to more than one biopsy. If the colposcopic examination showed no lesion in the cervix, a random biopsy specimen was obtained at the squamocolumnar junction in that quadrant at 3, 6, 9, or 12 o'clock. An endocervical curettage was performed after the cervical biopsy.

All biopsy specimens were fixed in formaldehyde and embedded in paraffin according to the routine procedures of the pathology department. The biopsy specimens were examined by the pathologist. After examining the specimen, an experienced expert pathologist made a final diagnosis according to the 2012 Lower Anogenital Squamous Project's two-tier squamous intraepithelial lesions (SIL) terminology (LSIL and HSIL). In uncertain cases, P16 immunohistochemistry should be used in the assessment of HSILs, particularly in dealing with a specimen that already has an $\mathrm{H} \& \mathrm{E}$ morphologic interpretation of HSIL(CIN 2) and in the distinction between HSIL(CIN2 or CIN3) and its mimics, such as immature squamous metaplasia, atrophy, reparative epithelial changes, and tangential cutting [7]. LSIL refers to koilocytosis, flat condyloma, CIN 1, or P16 negative CIN2; HSIL refers to CIN3 or P16 positive CIN2 [8]. A pathology slide review was not performed.

\section{Statistical methods}

SPSS 20.0 software (SPSS, Inc., Chicago, IL) was used for statistical analysis. The significance of agreement between the colposcopic diagnosis and cervical pathology was assessed with the chi-square statistic ( $\chi^{2}$ test) and weighted kappa values. The sensitivity, specificity, PPV, NPV, and Youden index were used to compare the colposcopic diagnosis and cervical pathology. P-values < 0.05 were considered statistically significant.

\section{Abbreviations}

HSIL: high-grade squamous intraepithelial lesion; IFCPC: International Federation for Cervical Pathology and Colposcopy; LSIL: low-grade squamous intraepithelial lesion; MIC: micro invasive cervical cancer; NPV: negative predictive value; PPV: positive predictive value; RCI: Reid colposcopic index; TZ: transformation zone; ASCCP: American Society for Colposcopy and Cervical Pathology

\section{Author contributions}

Aiping Fan: data collection, manuscript writing, project development; Chen Wang: data collection, manuscript writing; Liqin Zhang: data collection and analysis; Ye Yan: data analysis; Cha Han: data collection; Fengxia Xue: project development, manuscript revision.

\section{ACKNOWLEDGMENTS}

We thank Dr. Tao Shi of the Department of Pathology for Tianjin Medical University General Hospital for his help in the data collection.

\section{CONFLICTS OF INTEREST}

There are no conflicts of interest to declare.

\section{FUNDING}

This study was supported by the National Natural Science Foundation of China (No. 81471419).

\section{REFERENCES}

1. Reid R, Scalzi P. Genital warts and cervical cancer. VII. An improved colposcopic index for differentiating benign papilloma viral infections from high-grade cervical intraepithelial neoplasia. Am J Obstet Gynecol. 1985; 153:611-618.

2. Apgar BS, Kaufman AJ, Bettcher C, Parker-Featherstone E. Gynecologic procedures: colposcopy, treatments 
for cervical intraepithelial neoplasia and endometrial assessment. Am Fam Physician. 2013; 87:836-843.

3. Massad LS, Collins YC. Strength of correlations between colposcopic impression and biopsy histology. Gynecol Oncol. 2003; 89:424-428.

4. Baum ME, Rader JS, Gibb RK, McAlister RP, Powell MA, Mutch DG, Gao F, Wright JD. Colposcopic accuracy of obstetrics and gynecology residents. Gynecol Oncol. 2006; 103:966-970.

5. Moss EL, Hadden P, Douce G, Jones PW, Arbyn M, Redman CW. Is the colpscopically-directed punch biopsy a reliable diagnositc test in women with minor cytological lesions? J Low Genit Tract Disease. 2012; 16:421-426.

6. Bornstein J, Bentley J, Bösze P, Girardi F, Haefner H, Menton M, Perrotta M, Prendiville W, Russell P, Sideri M, Strander B, Tatti S, Torne A, et al. 2011 colposcopic terminology of the International Federation for Cervical Pathology and Colposcopy. Obstet Gynecol. 2012; 120:166-172.

7. Darragh TM, Colgan TJ, Cox JT, Heller DS, Henry MR, Luff RD, McCalmont T, Nayar R, Palefsky JM, Stoler MH, Wilkinson EJ, Zaino RJ, Wilbur DC; Members of LAST Project Work Groups. The Lower Anogenital Squamous Terminology Standardization Project for HPV-Associated Lesions: background and consensus recommendations from the College of American Pathologists and the American Society for Colposcopy and Cervical Pathology. J Lower Genit Tract Dis. 2012; 16:205-242.

8. Kurman RJ, Carcangiu ML, Herrington CS, Young RH. WHO classification of tumors of female reproductive organs. 4th Edition. Lyon: International Agency for Research on Cancer (IARC); 2014:172-176.

9. Hong DG, Seong WJ, Kim SY, Lee YS, Cho YL. Prediction of high-grade squamous intraepithelial lesion using the modified Reid index. Int J Clin Oncol. 2010; 15:65-69.

10. Bowring J, Strander B, Young M, Evans H, Walker P. The Swede score: evaluation of a scoring system designed to improve the predictive value of colposcopy. J Low Genit Tract Dis. 2010; 14:301-305.

11. Mousavi AS, Fakour F, Gilani MM, Behtash N, Ghaemmaghami F, Karimi Zarchi M. A prospective study to evaluate the correlation between Reid colposcopic index impression and biopsy histology. J Low Genit Tract Dis. 2007; 11:147-150.

12. Nazeer S, Shafi MI. Objective perspective in colposcopy. Best Pract Res Clin Obstet Gynaecol. 2011; 25:631-640.

13. Tatiyachonwiphut $\mathrm{M}$, Jaishuen A, Sangkarat $\mathrm{S}$, Laiwejpithaya S, Wongtiraporn W, Inthasorn P, Viriyapak B, Warnnissorn M. Agreement between colposcopic diagnosis and cervical pathology: siriraj hospital experience. Asian Pac J Cancer Prev. 2014; 15:423-426.

14. Li Y, Zhang H, Zheng R, Xie F, Sui L. Agreement between colposcopic diagnosis with 2011 international terminology of colposcopy and cervical in cervical lesions (abstract).
[Article in Chinese]. Zhonghua Fu Chan Ke Za Zhi. 2015; 50:361-366.

15. Durdi GS, Sherigar BY, Dalal AM, Desai BR, Malur PR. Correlation of colposcopy using Reid colposcopic index with histopathology- a prospective study. J Turk Ger Gynecol Assoc. 2009; 10:205-207.

16. Massad LS, Jeronimo J, Katki HA, Schiffman M; National Institutes of National Institutes of Health/American Society for Colposcopy and Cervical Pathology Research Group. The accuracy of colposcopic grading for detection of highgrade cervical intraepithelial neoplasia. J Low Genit Tract Dis. 2009; 13:137-144.

17. Hammes LS, Naud P, Passos EP, Matos J, Brouwers K, Rivoire W, Syrjänen KJ. Value of the international Federation for Cervical Pathology and Colposcopy (FICPC) Terminology in predicting cervical disease. J Low Genit Tract Dis. 2007; 11:158-165.

18. Luyten A, Buttmann-Schweiger N, Hagemann I, Scherbring S, Boehmer G, Gieseking F, Woelber L, Glasenapp F, Hampl M, Kuehler-Obbarius C, van den Bergh M, Petry KU; German Colposcopy Network (G-CONE) and the German Colposcopy Study Group. Utility and Reproducibility of the International Federation for Cervical Pathology and Colposcopy Classification of Transformation Zones in Daily Practice: a multicenter study of the German Colposcopy Network. J Low Genit Tract Dis. 2015; 19:185-188.

19. Wentzensen N, Massad LS, Mayeaux EJ Jr, Khan MJ, Waxman AG, Einstein MH, Conageski C, Schiffman MH, Gold MA, Apgar BS, Chelmow D, Choma KK, Darragh TM, et al. Evidence-based consensus recommendations for practice for cervical cancer prevention in the United States. J Low Genit Tract Dis. 2017; 21:216-222.

20. Kierkegaard O, Byralsen C, Hansen KC, Frandsen KH, Frydenberg M. Association between colposcopic findings and histology in cervical lesions: the significance of the sizeof the lesion. Gynecol Oncol. 1995; 5766-71

21. Munmany M, Marimon L, Cardona M, Nonell R, Juiz M, Astudillo R, Ordi J, Torné A, Del Pino M. Small lesion size measured by colposcopy may predict absence of cervical intraepithelial neoplasia in a large loop excision of the transformation zone specimen. BJOG 2017; 124:495-502.

22. Scheungraber C, Glutig K, Fechtel B, Kuehne-Heid R, Duerst M, Schneider A. Inner border -- a specific and significant colposcopic sign for moderate or severe dysplasia (cervical intraepithelial neoplasia 2 or 3). J Lower Genit Tract Dis. 2009; 13:1-4.

23. Vercellino GF, Erdemoglu E, Chiantera V, Vasiljeva K, Drechsler I, Cichon G, Schneider A, Böhmer G. Validity of the colposcopic criteria inner border sign, ridge sign, and rag sign for detection of high-grade cervical intraepithelial neoplasia. Obstet Gynecol. 2013; 121:624-631.

24. Vercellino GF, Erdemoglu E, Chiantera V, Vasiljeva K, Malak AH, Schneider A, Böhmer G. Clinical relevance 
of objectifying colposcopy. Arch Gynecol Obstet. 2015; 291:907-915.

25. Schneider A, Rakozy C, Stolte C, Bothur-Schäfer P, Rothe H, Welcker T, Choly N, Roesgen A, Böhmer G. Correlation between VITOM $^{(\circledR)}$ videocolposcopy and histopathology for pathognomonic grading criteria. Arch Gynecol Obstet. 2015; 292:1361-1366.
26. Yang B, Pretorius RG, Belinson JL, Zhang X, Burchette R, Qiao YL. False negative colposcopy is associated with thinner cervical intraepithelial neoplasia 2 and 3. Gynecol Oncol. 2008; 110:32-36. 